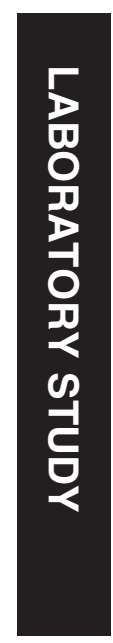

${ }^{1}$ Eye Clinic and Institute for Ophthalmic Research, Centre for Ophthalmology, University of Tübingen, Tübingen, Germany

${ }^{2}$ Department of Ophthalmology, LudwigMaximilians-University, Munich, Germany

${ }^{3} 1$ st Eye Hospital, Medical University of Lublin, Lublin, Poland

${ }^{4}$ Department of Anatomy, Medical Faculty 'Carl Gustav Carus', Technical University of Dresden, Dresden, Germany

${ }^{5}$ Department of Chemistry, Ludwig-MaximiliansUniversity, Munich, Germany

Correspondence: S Thaler, Eye Clinic and Institute for Ophthalmic Research, Centre for Ophthalmology, University of Tübingen, Schleichstrasse 12-16, D-72076 Tübingen, Germany

Tel: +49 707129837 21; Fax: +497071295018. E-mail: sebastian.thaler@ uni-tuebingen.de

Received: 26 February 2014 Accepted in revised form:

2 October 2014

Published online:

19 December 2014

\section{In vivo biocompatibility of a new cyanine dye for ILM peeling}

\begin{abstract}
Purpose To investigate the biocompatibility of the new cyanine dye: 3,3'-Di-(4sulfobutyl)-1,1,1', $\mathbf{1}^{\prime}$-tetramethyl-di-1Hbenz[e]indocarbocyanine (DSS) as a vital dye for intraocular application in an in vivo rat model and to evaluate the effects of this dye on retinal structure and function.

Methods DSS at a concentration of $\mathbf{0 . 5 \%}$ was applied via intravitreal injections to adult Brown Norway rats with BSS serving as a control. Retinal toxicity was assessed 7 days later by means of retinal ganglion cell (RGC) counts, light microscopy, optical coherence tomography (OCT), and electroretinography (ERG).

Results No significant decrease in RGC numbers was observed. No structural changes of the central retina were observed either in vivo (OCT) or under light microscopy. ERGs detected a temporary reduction of retinal function 7 days after injection; this was no longer evident 14 days after injection. Conclusions DSS showed good biocompatibility in a well-established experimental in vivo setting and may be usable for intraocular surgery as an alternative to other cyanine dyes. In contrast to indocyanine green, it additionally offers fluorescence in the visual spectrum. Further studies with other animal models are needed before translation into clinical application. Eye (2015) 29, 428-435; doi:10.1038/eye.2014.295; published online 19 December 2014
\end{abstract}

\section{Introduction}

Vital dyes have become widely used in vitreoretinal surgery for visualizing semitransparent structures such as the internal limiting membrane (ILM). Currently, indocyanine green (ICG), a cyanine dye, and brilliant blue $\mathrm{G}$ (BBG), an anionic triarylmethane
S Thaler ${ }^{1}$, C Haritoglou' ${ }^{2}$, F Schuettauf ${ }^{1}$, T Choragiewicz ${ }^{1,3}$, CA May ${ }^{4}$, F Gekeler ${ }^{1}$, MD Fischer ${ }^{1}, \mathrm{H}$ Langhals $^{5}$ and A Schatz ${ }^{1}$ dye, are most frequently used for ILM peeling. Such vital dyes facilitate the removal of the ILM by the surgeon, and thus can probably prevent the damage to underlying retinal structures.

However, the possible toxicity of these vital dyes has been discussed since the introduction of ICG to vitreoretinal surgery about 10 years ago. Several in vitro and in vivo biocompatibility studies have found retinotoxic effects of ICG, ${ }^{1-3}$ whereas others have not. ${ }^{4,5}$ The discussion about ICG's safety has also led to the recommendations regarding concentrations, exposure times, $\mathrm{pH}$, illumination, and other factors. 6,7

The vital dye BBG was introduced as an alternative to ICG. ${ }^{8}$ Overall, it exhibited a better biocompatibility profile than ICG in many in vitro and in vivo studies. ${ }^{9-11}$ On the other hand, randomized clinical studies providing evidence that clinical outcomes are better with BBG than with ICG in ILM-peeling procedures are still lacking. Importantly, the staining properties of ICG seem superior to those of $\mathrm{BBG}^{12}$ - a possible reason for the former's continued widespread use.

Our group accordingly undertook to design alternative cyanine dyes with the goal of finding a dye with the good contrasting characteristics of ICG but even better biocompatibility. One of these new dyes, 3,3'-Di-(4-sulfobutyl)-1,1,1', 1'-tetramethyl-di-1H-benz[e]indocarbocyanine (DSS) showed good in vitro biocompatibility with different cell lines and good staining properties at concentrations of 0.25 or $0.5 \% .^{13}$

In the present study, we therefore evaluated the biocompatibility of DSS in a well-established in vivo rat model using a previously described combined morphological and functional approach. ${ }^{14}$

\section{Materials and methods}

Dye preparation

DSS was synthesized as described in detail previously. ${ }^{13}$ A stock solution of DSS was 
prepared by dissolving DSS in distilled water and by adding an equal volume of sodium chloride solution (620 mosm) to gain an osmolarity of 308 mosm (as in balanced salt solution (BSS)). The stock solution was then diluted to $0.5 \%$ DSS using BSS.

\section{Animals}

All experiments were performed in compliance with the guidelines for animal care in the European Community and those of the Association for Research in Vision and Ophthalmology. Female Brown Norway rats (Charles River, Sulzfeld, Germany) with a body weight of 125-160 g were used. Rats were housed under a 12-h light-dark cycle.

\section{Intravitreal injections}

Rats were anesthetized with chloral hydrate $7 \%(6 \mathrm{ml} / \mathrm{kg}$ body weight) by intraperitoneal injections. Intravitreal injections were performed using a heat-pulled glass capillary connected to a microsyringe (Drummond Scientific Co., Broomall, PA, USA) under direct observation through the microscope. Eyes with lens damage were excluded from the experiments and not used thereafter.

For retinal ganglion cell (RGC) quantification, histology, ERG, and optical coherence tomography (OCT), single injections of $2 \mu \mathrm{l}$ of DSS in a concentration of $0.5 \%$ as well as BSS were administered intravitreally into the posterior bulbus as follows: left eyes were injected with DSS, while the contralateral eyes served as control eyes and received only BSS. Untreated eyes served as additional controls for in vivo imaging by OCT and subsequent histology. The final intraocular concentration of DSS was $0.05 \%$ in an average rat eye (estimated intravitreal volume: $20 \mu \mathrm{l}$ ).

\section{Retrograde labelling and quantification of RGC}

RGC survival (7 rats per group, dye concentration $0.5 \%$ ) was assessed as described previously. ${ }^{15}$ Five days after intravitreal injections the anaesthetized rats were placed in a stereotaxic frame (TSE Systems, Bad Homburg, Germany). A portion of the skull and the meninges were removed, exposing the neocortex overlying the tectum on the side contralateral to the lesioned eye. Three injections of a fluorescent tracer (hydroxystilbamidine methanesulfonate; Molecular Probes, Eugene, OR, USA) were administered over a period of $2 \mathrm{~min}$, each targeting all layers of both the superior colliculi. Two days later the animals were sacrificed with choral hydrate. The cornea of each eye was marked at the 12 o'clock position for orientation in retinal quadrants. The eyes were then enucleated and the retinas were dissected, flat-mounted, and fixed in 3\% paraformaldehyde for $30 \mathrm{~min}$.

Visualization was performed immediately under a fluorescence microscope. RGC were counted in 12 distinct areas of $62500 \mu \mathrm{m}^{2}$, as described in detail previously. ${ }^{16}$ Images obtained using a digital imaging system (ImagePro 3.0, Media Cybernetics Inc., Silver Spring, MD, USA) connected to a microscope were coded and analysed in a masked manner. Labelled cells were defined as surviving. Counts are expressed in cell density $\left(=\right.$ cells $\left./ \mathrm{mm}^{2}\right) \pm$ SEM.

Statistical analysis was performed using GraphPad Prism version 5.04 for Windows (GraphPad Software, San Diego, CA, USA). A paired, two-tailed Student's $t$-test was used for data analysis. Differences were considered significant when $P<0.05$.

\section{Histology}

One week after intravitreal injections (DSS at 0.5\%, BSS as controls, $n=3$ rats per group), the eyes were enucleated and immediately immersion-fixed in Ito's solution (2.5\% glutaraldehyde, $2.5 \%$ paraformaldehyde, $0.01 \%$ picric acid in $0.1 \mathrm{M}$ cacodylate buffer, $\mathrm{pH} 7.2$ ).

The eyes were bisected into nasal and temporal segments. The specimens were post-fixed in $1 \%$ osmium tetroxide, rinsed in cacodylate buffer, dehydrated in an ascending series of alcohols, and embedded in araldite. Semi-thin sections were cut along a superior-inferior plane, stained with toluidine blue, and investigated by light microscopy. In addition, sections were taken from the injection side.

The total thickness of the neural retina and the thicknesses of the different retinal layers were quantitatively evaluated in an area lying $200 \mu \mathrm{m}$ from the optic nerve head rim, and single-thickness measurements were obtained within the next $300 \mu \mathrm{m}$ peripherally. As there was no statistical difference between the superior and the inferior regions, the data of each individual eye were pooled (using one mean value for each individual eye). Statistical analysis was performed using the Wilcoxon test (with $\alpha=0.01$ ).

\section{Electroretinography}

Electrophysiological measurements were performed at baseline (before intravitreal injections) and at 7 and 14 days after intravitreal injections of both the eyes (BSS and DSS; $n=9$ ).

After dark adaptation for $12 \mathrm{~h}$, rats were anaesthetized under dim red illumination with a combination of intraperitoneally applied ketamine $(100 \mathrm{mg} / \mathrm{kg})$ and xylazine $(5 \mathrm{mg} / \mathrm{kg})$. The core body temperature of each animal was monitored and kept at $36 \pm 1{ }^{\circ} \mathrm{C}$ using a 
heating plate. Electrodes were placed as described previously. ${ }^{17}$ Ten minutes before starting ERG measurements, the pupils were fully dilated with tropicamide and the dim red light was turned off.

ERGs were recorded and stored for offline analysis using the Espion (Diagnosys LLC, Cambridge, UK) system. The recorded signal was filtered online with a band-pass filter from 0.3 to $300 \mathrm{~Hz}$. Electrode impedance was checked before and after each measurement in each animal using a built-in algorithm, and was $<10 \mathrm{k} \Omega$ at $25 \mathrm{~Hz}$ (manufacturer's recommendation). The eyes were stimulated using a Ganzfeld stimulator (ColorDome; Diagnosys LLC).

Under dark-adapted conditions, light stimuli (4 ms duration) with increasing luminance in 12 steps $(0.000003$ to $3 \mathrm{~cd} . \mathrm{s} / \mathrm{m}^{2}$ ) were used to elicit responses that ranged from a baseline (b-wave amplitude undetectable) to a saturated b-wave response. Results for the first three steps $\left(0.000003\right.$ to $\left.0.00003 \mathrm{~cd} . \mathrm{s} / \mathrm{m}^{2}\right)$ were taken as the average of 10-20 responses with an inter-stimulus interval (ISI) of $2-3 \mathrm{~s}$; for the next five steps $\left(0.0001\right.$ to $\left.0.01 \mathrm{~cd} . \mathrm{s} / \mathrm{m}^{2}\right), 3-5$ responses with an ISI of 5-10 s were averaged; and for the last four steps $\left(0.03\right.$ to $\left.3 \mathrm{~cd} . \mathrm{s} / \mathrm{m}^{2}\right)$, averages of 2 responses with ISI of 30-45 s were determined.

A hyperbolic saturation model Naka ${ }^{18}$ was fitted for the interrelation between b-wave amplitude and luminance (I) in order to derive two parameters: $V_{\max }$ as the saturated $b$-wave amplitude; $k$ as the luminance required for the b-wave to reach $1 / 2$ of $V_{\max }$ (semisaturation point), with $n$ as non-dimensional parameter related to the linear phase slope.

$V(I)=\frac{\operatorname{Vmax} \times I^{n}}{I^{n} \times k^{n}}$

Oscillatory potentials (OPs) were obtained from the response elicited by the flash of $3 \mathrm{~cd}$.s $/ \mathrm{m}^{2}$ by means of a fast Fourier transformation implemented as a band-pass frequency filter (from 75 to $300 \mathrm{~Hz}$ ). The absolute value of the area under the curve for all OP wavelets was determined between a- and b-wave implicit times.

The eyes were light adapted for $10 \mathrm{~min}$ inside the Ganzfeld with a background illumination of $30 \mathrm{~cd} / \mathrm{m}^{2}$ (white-6500 K) for rod saturation. Light-adapted recordings were performed with single-flash stimuli and $20 \mathrm{~Hz}$ flicker stimulation (both with $3 \mathrm{~cd} . \mathrm{s} / \mathrm{m}^{2}$ ). The average of 20 responses with an ISI of $2 \mathrm{~s}$ was determined for single-flash responses. For flicker stimulation the average of 25-30 responses was determined.

The results of both eyes were compared at baseline examination using a paired, two-tailed Student's $t$-test, and no significant difference was ascertained between the right and left eyes ( $P=0.19-0.86$; data not shown). An intra-individual quotient (ratio) was calculated between the results found for both eyes at baseline and at days 7 and 14 after injection. These ratios were compared between the BSS-injected eyes (right; $n=9$ ) and the DSS-injected eyes (left; $n=9$ ) using a paired, two-tailed Student's $t$-test with a significance level of $P=0.05$ and the Bonferroni-Holm's correction for multiple comparisons.

\section{In vivo retinal imaging}

For non-invasive retinal imaging a Spectralis HRA + OCT device (Heidelberg Engineering, Heidelberg, Germany) was used for confocal scanning laser ophthalmoscopy (cSLO) and spectral domain OCT (SD-OCT) as previously described. ${ }^{19}$ Both vertical and horizontal SD-OCT cross-sections centred on the optic disc were recorded to ensure perpendicular orientation of retinal tissue. Quantification of central retinal thickness based on high-resolution vertical line scans (superior retina, optic nerve head, and inferior retina) was performed using the proprietary software from Heidelberg Engineering (Eye Explorer version 1.6.4.0, HRA/Spectralis Viewing Module version 5.3.2.0). Total retinal thickness (TRT) was quantified by 1536 consecutive A-Scans using a previously published ImageJ plug-in (http:/ / imagej.nih.gov/ij/plugins/ heyex/index.html) as previously described by Knott et al. ${ }^{20}$ All measurements were taken by one examiner who was blind to treatment conditions. For statistical analysis, TRT values were interpolated at 100 A-Scan increments along a vertical meridian in both eyes at three time points $(n=9)$. All results are expressed as mean $\pm \mathrm{SD}$. Comparisons between treated and untreated eyes were made by paired, two-tailed Student's $t$-test for SD-OCT data. A $P$-value of $<0.05$ was considered statistically significant.

\section{Results}

\section{In vivo retinal structure}

In vivo cSLO and OCT imaging of the retinas showed essentially unchanged anatomy during the time span investigated. TRT remained normal in DSS-injected eyes $\left(\mathrm{TRT}_{\mathrm{DSS}} ; n=9\right)$ compared with BSS-injected eyes (TRT $\mathrm{BSS}_{\text {; }}$ $n=9)$ on both day $7\left(\mathrm{TRT}_{\mathrm{DSS}}=191.75 \pm 11.67 \mathrm{vs}\right.$ $\left.\mathrm{TRT}_{\mathrm{BSS}}=192.95 \pm 12.23\right)$ and day $14\left(\mathrm{TRT}_{\mathrm{DSS}}=173.80 \pm 9.02\right.$ vs $\left.\mathrm{TRT}_{\mathrm{BSS}}=176.34 \pm 9.55\right)$ after intravitreal application. Student's $t$-test showed no statistically significant differences in TRT values at any time.

\section{RGC numbers}

RGC numbers were assessed 7 days after intravitreal injections of DSS $0.5 \%$ and compared with BSS-injected controls (Figures 1 and 2). There was no significant 
difference in RGC numbers between the DSS and BSS eyes $(2467 \pm 157 ; 2654 \pm 139.5, n=7, P=0.3965)$. The BSS eyes showed no differences from untreated eyes (data not shown).

\section{Histology}

No histological retinal changes were detectable after injections of DSS $0.5 \%$ in comparison to BSS-injected controls. Normal retinal structures remained intact in all analysed retinas, and no signs of inflammation or retinal degeneration were visible. Quantitative analysis of the thickness of all retinal layers showed no statistically significant differences for any eye (Table 1).

\section{Electroretinography}

The following absolute data are presented as mean \pm SD.

Seven days after injection, $V_{\max }$ of the BSS-injected eyes was $657 \pm 75 \mu \mathrm{V}$ and $578 \pm 70 \mu \mathrm{V}$ in eyes injected with

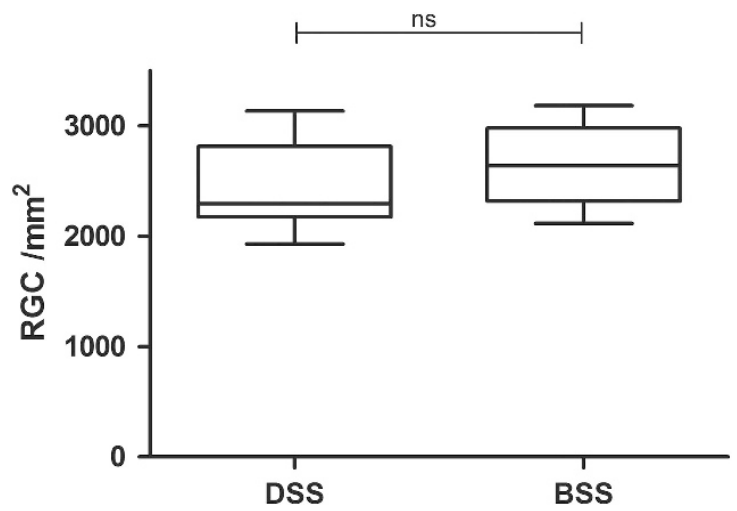

Figure 1 RGC density. RGC density in DSS-treated retinas 7 days after injection compared with BSS controls. The line in the box represents the median observation, the top and bottom boundaries of the box represent the 25 th and 75 th percentiles, and the whiskers extend to the outermost data points. ns, not significant.
DSS; 14 days after injection it was $597 \pm 101 \mu \mathrm{V}$ in BSS eyes and $569 \pm 62 \mu \mathrm{V}$ in DSS eyes. No statistically significant differences were found between BSS and DSS eyes at 7 days after injection for $V_{\max }(P=0.33$; Figure 3$)$ and for $k(P=0.92)$. For both parameters no significant differences were observed at day 14 after injection $\left(V_{\max }\right.$, $P=0.59 ; k, P=1.00)$

The dark-adapted b-wave amplitude of the standard flash using $3 \mathrm{~cd}$.s $/ \mathrm{m}^{2}$ at day 7 was $898 \pm 94 \mu \mathrm{V}$ in BSSinjected eyes and $802 \pm 95 \mu \mathrm{V}$ in DSS-injected eyes. At day 14 , it was $807 \pm 139 \mu \mathrm{V}$ in BSS-injected eyes and $775 \pm 87 \mu \mathrm{V}$ in DSS-injected eyes. No significant differences in b-wave amplitudes between both eyes were observed at day $7(P=0.25)$ and $14(P=0.76)$. No significant differences were found for implicit times (7 days, $P=0.69 ; 14$ days, $P=1.000)$. OPs in both eyes were not significantly different at day $7(P=0.10$; BSS,

$2014 \pm 274 \mu \mathrm{V} . \mathrm{ms}$ and DSS, $1813 \pm 201 \mu \mathrm{V}$.ms) and at day $14(P=1.00$; BSS, $1662 \pm 429 \mu$ V.ms and DSS,

$1581 \pm 404 \mu$ V.ms).

For light-adapted b-wave amplitudes of single-flash responses $\left(3 \mathrm{~cd} . \mathrm{s} / \mathrm{m}^{2}\right)$ significant differences were found at day $7(P=0.02$; Figure 3$)$, but no significant differences were found for day $14(P=0.38$; Figure 3$)$. In eyes injected with BSS, the b-wave amplitudes were $104 \pm 17 \mu \mathrm{V}$ at day 7 and $99 \pm 18 \mu \mathrm{V}$ at day 14 . DSS-injected eyes revealed b-wave amplitudes of $86 \pm 18 \mu \mathrm{V}$ at day 7 and $95 \pm 11 \mu \mathrm{V}$ at day 14 . No significant differences were found for implicit times at day 7 and 14 (day 7 , $P=0.61$; day $14, P=0.79$ ).

No statistically significant differences were found among ratios calculated between a-wave amplitudes, a-wave implicit times, a-wave slopes, and light-adapted flicker responses $(P=0.06-1.00)$.

\section{Discussion}

The present study investigated the in vivo biocompatibility of an alternative dye (DSS) for
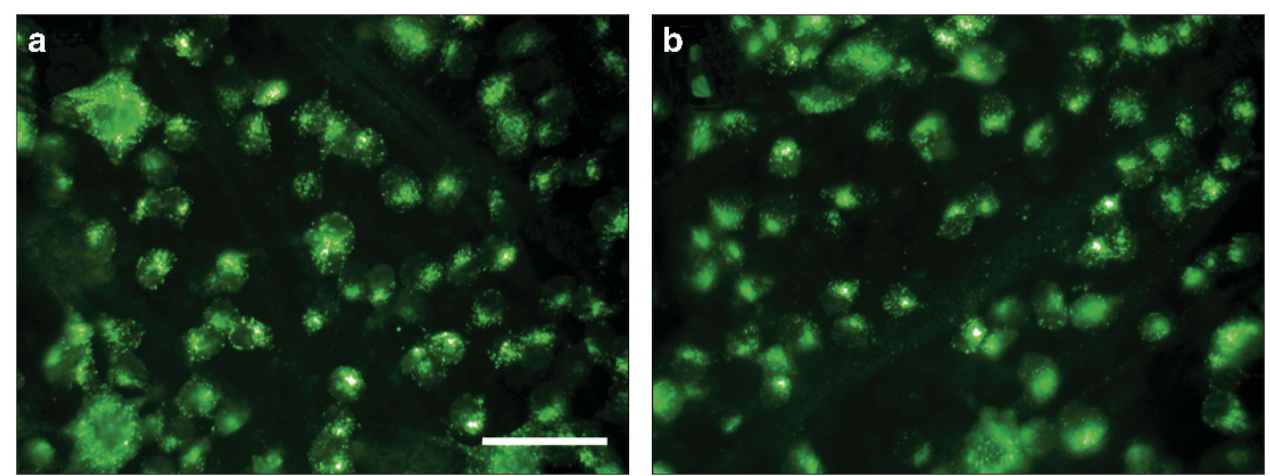

Figure 2 Retinal flat mounts. RGC: hydroxystilbamidine methanesulfonate back-labelled RGCs photographed 7 days after intravitreal injections of BSS (controls) (a) and DSS (b). Scale bar, $50 \mu \mathrm{m}$. 
Table 1 Mean thickness of different retinal layers

\begin{tabular}{lcc}
\hline Mean thickness in $\mu m$ & DSS $0.5 \%$ & BSS (controls) \\
\hline Total retinal thickness & $211 \pm 10$ & $215 \pm 13$ \\
NFL & $5.6 \pm 1.1$ & $6.0 \pm 1.6$ \\
IPL & $61 \pm 6$ & $59 \pm 5$ \\
OPL & $8.4 \pm 1.5$ & $8.3 \pm 1 ., 2$ \\
\hline
\end{tabular}

Abbreviations: BSS, balanced salt solution; DSS, 3,3'-Di-(4-sulfobutyl)1,1,1', $1^{\prime}$-tetramethyl-di-1H-benz[e]indo-carbocyanine; IPL, inner plexiform layer; NFL, nerve fibre layer; OPL, outer plexiform layer. Thicknesses are similar in DSS-injected eyes and BSS-injected control eyes $(n=3)$

visualizing the ILM in chromovitrectomy. Currently, the cyanine dye ICG and the triarylmethane dye BBG are principally used for ILM staining in clinical routine. Staining characteristics and modification of ILM stiffness (allowing for easier ILM peeling) seem to be superior for ICG compared with BBG. ${ }^{21}$ However, BBG has shown a better biocompatibility in many in vitro and in vivo studies, $, 9,10,22,23$ as well as a possibly better clinical outcome in some small non-randomized clinical studies. $^{24,25}$ Therefore, we developed DSS, an alternative cyanine dye, with the goal of gaining good staining properties, additional fluorescence, and better biocompatibility than that of ICG.

In a previous in vitro study, DSS showed markedly better biocompatibility compared with ICG and led to strong staining of the ILM at concentrations of 0.25 and $0.5 \%$, allowing for safe ILM peeling. In addition, both the absorption spectrum and the fluorescence spectrum of DSS are in the visible range of the human eye, in contrast to those of ICG. ${ }^{13}$

A well-established experimental setting with a rat model was used in the present in vivo study. ${ }^{14,17} \mathrm{We}$ chose to test DSS at a concentration of $0.5 \%$. This had no adverse effects in different cell lines for up to $120 \mathrm{~s}$ of exposition time and was the highest concentration used in vitro for optimal staining of the ILM. ${ }^{13}$ Owing to the good biocompatibility of DSS at $0.5 \%$ in the present study, lower concentrations were not tested. Importantly, this concentration also afforded optimum comparability with previously performed toxicity studies of ICG in the same animal model. ${ }^{17,26}$

Assessment of RGC numbers after retrograde labelling with a fluorescent marker is a sensitive tool for evaluating the loss of functional RGC in vivo. ${ }^{27-30}$ In addition to analysis of retinal layer thicknesses, it allows convenient and very reliable direct evaluation of RGC damage. Composing as they do the innermost retinal cell layer, the RGCs are especially affected by intravitreally applied dyes for ILM peeling. In a previous in vivo study, the application of $0.5 \%$ ICG (but not lower concentrations) resulted in significant changes of the inner retinal layers under light microscopy.
Notably, RGC numbers were already reduced at an ICG concentration of $0.0002 \% .{ }^{26}$ In a study of the biocompatibility of Rhodamine 6G (R6G), another fluorescent candidate dye for ILM peeling, light microscopy showed no changes in the central retina, but RGC loss in the central retina was significant under 0.2 and $0.5 \%$ of R6G. ${ }^{14}$ In the present study, DSS at $0.5 \%$ caused no significant RGC loss compared with BSS, a result comparable with those previously found for the triarylmethane dye BBG. ${ }^{11}$

Neither light microscopic analysis of retinal slices nor in vivo cSLO and OCT imaging of the retina showed significant anatomical changes of retinal structure in DSS-treated eyes.

Importantly, functional ERG analysis of the retina revealed a reversible b-wave reduction after 7 days in DSS-treated eyes that was no longer measurable after 14 days. Similar transient ERG changes have been previously described for other intraocular vital dyes. ${ }^{31}$ Our results therefore indicate that DSS is followed by reversible functional changes, but no structural damage after retinal exposure. In contrast, a previous study with ICG $0.5 \%$ in the same model showed a much more pronounced $b$-wave reduction that remained significant even 14 days after ICG application. ${ }^{17}$ This accords with the results of other ERG studies ${ }^{3}$ in which b-wave reduction was significant even after 2 months of dye exposure, and the retinal structure was altered. Other studies found significant $b$-wave reductions for ICG $0.5 \%$; these persisted for $<4$ weeks but were accompanied by anatomical changes. ${ }^{32}$

Our results for DSS biocompatibility with a view to potential clinical use must be interpreted with caution inasmuch as animal experiments may fail to reflect important, potentially adverse effects in a clinical situation. In particular, the following systematic limitations of our experimental setting must be kept in mind: first, the dye was injected into nonvitrectomized eyes in contrast to the clinical situation in ILM peeling. Clinically, therefore the dye concentration at the central retina might be higher than that in our study. However, ILM staining with DSS was already sufficient with half the dose tested in vitro (0.25\% DSS). Furthermore, the dye exposure time in our setting was much longer (7-14 days) than in the clinical situation, in which washout usually takes place about 1 min after dye exposure.

The advantage of our setting is its good comparability to previous in vivo studies with ICG or BBG. In addition, the absence of toxic effects after such a long exposure reduces the probability of toxic effects after shorter exposure times.

The relatively short assessment time of 14 days in our study does not allow an evaluation of possible long-term 


\section{Vmax of the scotopic fit}
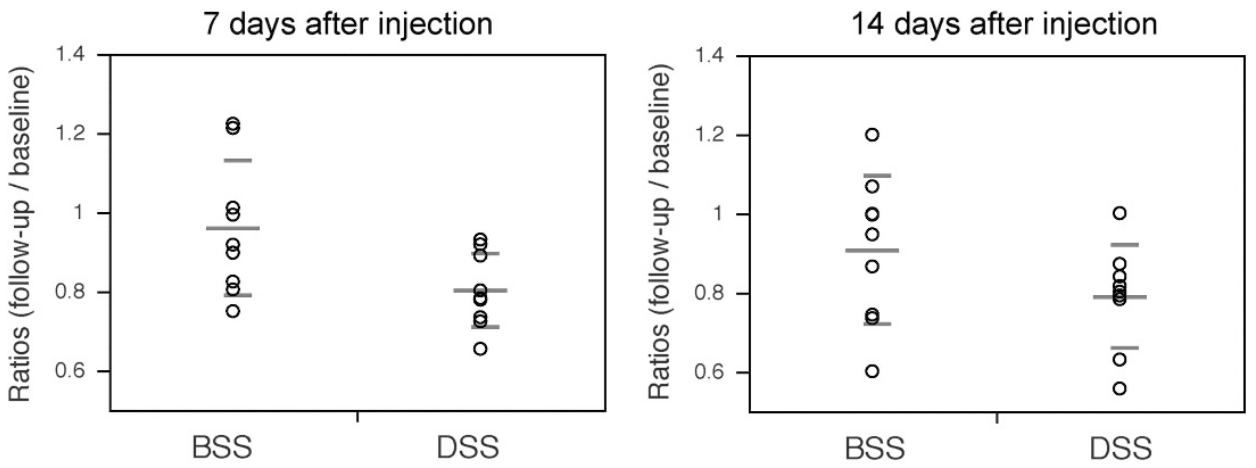

light-adapted $3 \mathrm{~cd} . \mathrm{s} / \mathrm{m}^{2}$ b-wave amplitude
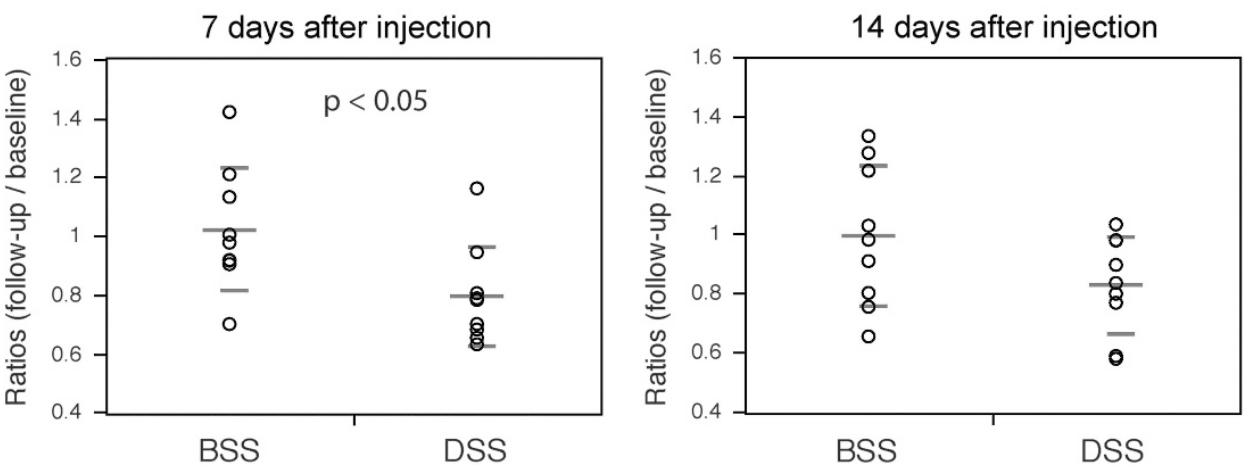

Figure 3 ERGs. Analyses of intra-individual ratios (follow-up/baseline) show significant reductions of amplitudes 7 days after injection in DSS eyes compared with BSS eyes for the light-adapted b-wave amplitudes $\left(3 \mathrm{~cd} \mathrm{~s} / \mathrm{m}^{2}\right)$, but no significant differences for $V_{\max }$ of the scotopic fit. No significant differences were observable on day 14 after treatment. The whiskers indicate means and SD.

effects. For example, a long-term intraocular presence despite washout has been postulated for some dyes such as ICG. ${ }^{33}$

Up to now, the search for alternative ILM stains has focused mainly on absorption qualities and nonfluorescent dyes. ${ }^{26,34,35}$ However, fluorescence represents a new, interesting approach in view of dose reduction, better visibility, and possible advantages of combined dyes analogous to, for example, MembraneBlue Dual.

Previously tested fluorescent dyes such as R6G have lacked good ocular biocompatibility. ${ }^{14}$ To our knowledge, DSS is the first ILM-staining dye with fluorescence in the visible spectrum that has been tested for intraocular use, and provides good staining abilities and good biocompatibility both in vitro and in vivo in ocular tissue. The results of our study indicate that DSS biocompatibility is superior to that of ICG in equivalent experimental settings.

In summary, our tests indicated that the cyanine dye DSS has a good potential for intraocular use, with interesting features, and combines the advantages of ICG (staining characteristics) and BBG (biocompatibility).
Further studies with other animal models will be needed to confirm these results before a possible transfer to clinical practice.

\section{Summary}

What was known before

- Vital dyes have become widely used in vitreoretinal surgery for visualizing semitransparent structures such as the internal limiting membrane. Related to staining properties, indocyanine green seems to be superior to other dyes in some respects; however, the issue of its safety for intraocular use is controversial.

What this study adds

- In vivo testing of a new alternative cyanine dye to indocyanine green with comparable contrasting characteristics but even better biocompatibility. In addition to indocyanine green-comparable staining properties, the new cyanine dye exhibits fluorescence in the visible spectrum.

\section{Conflict of interest}

The authors declare no conflict of interest. 


\section{Acknowledgements}

ST and FS received funding in the form of a grant from the Neuroophthalmologische Gesellschaft, Tübingen, Germany.

\section{References}

1 Sippy BD, Engelbrecht NE, Hubbard GB, Moriarty SE, Jiang S, Aaberg Jr TM et al. Indocyanine green effect on cultured human retinal pigment epithelial cells: implication for macular hole surgery. Am J Ophthalmol 2001; 132: 433-435.

2 Gandorfer A, Haritoglou C, Gandorfer A, Kampik A. Retinal damage from indocyanine green in experimental macular surgery. Invest Ophthalmol Vis Sci 2003; 44: 316-323.

3 Enaida H, Sakamoto T, Hisatomi T, Goto Y, Ishibashi T. Morphological and functional damage of the retina caused by intravitreous indocyanine green in rat eyes. Graefes Arch Clin Exp Ophthalmol 2002; 240: 209-213.

4 Grisanti S, Szurman P, Gelisken F, Aisenbrey S, Oficjalska-Mlynczak J, Bartz-Schmidt KU. Histological findings in experimental macular surgery with indocyanine green. Invest Ophthalmol Vis Sci 2004; 45: 282-286.

5 Kadonosono K, Takeuchi S, Yabuki K, Yamakawa T, Mekada A, Uchio E. Absorption of short wavelengths of endoillumination in indocyanine green solution: implications for internal limiting membrane removal. Graefes Arch Clin Exp Ophthalmol 2003; 241: 284-286.

6 Stanescu-Segall D, Jackson TL. Vital staining with indocyanine green: a review of the clinical and experimental studies relating to safety. Eye (Lond) 2009; 23: 504-518.

7 Thompson JT, Haritoglu C, Kampik A, Langhals H. Should Indocyanine green should be used to facilitate removal of the internal limiting membrane in macular hole surgery. Surv Ophthalmol 2009; 54: 135-138.

8 Enaida H, Hisatomi T, Hata Y, Ueno A, Goto Y, Yamada Tet al. Brilliant blue $\mathrm{G}$ selectively stains the internal limiting membrane/brilliant blue G-assisted membrane peeling. Retina 2006; 26: 631-636.

9 Kawahara S, Hata Y, Miura M, Kita T, Sengoku A, Nakao S et al. Intracellular events in retinal glial cells exposed to ICG and BBG. Invest Ophthalmol Vis Sci 2007; 48: 4426-4432.

10 Ueno A, Hisatomi T, Enaida H, Kagimoto T, Mochizuki Y, Goto Y et al. Biocompatibility of brilliant blue $\mathrm{G}$ in a rat model of subretinal injection. Retina 2007; 27: 499-504.

11 Remy M, Thaler S, Schumann RG, May CA, Fiedorowicz M, Schuettauf $\mathrm{F}$ et al. An in vivo evaluation of brilliant blue $\mathrm{G}$ in animals and humans. Br J Ophthalmol 2008; 92: 1142-1147.

12 Henrich PB, Priglinger SG, Haritoglou C, Josifova T, Ferreira PR, Strauss RW et al. Quantification of contrast recognizability during brilliant blue G- and indocyanine green-assisted chromovitrectomy. Invest Ophthalmol Vis Sci 2011; 52: 4345-4349.

13 Haritoglou C, Kernt M, Laubichler P, Langhals H, Eibl K, Varja A et al. Synthesis, staining properties, and biocompatibility of a new cyanine dye for ILM peeling. Graefes Arch Clin Exp Ophthalmol 2012; 250: 829-838.

14 Thaler S, Haritoglou C, Choragiewicz TJ, Messias A, Baryluk A, May CA et al. In vivo toxicity study of rhodamine $6 \mathrm{G}$ in the rat retina. Invest Ophthalmol Vis Sci 2008; 49: 2120-2126.

15 Schuettauf F, Eibl KH, Thaler S, Shinoda K, Rejdak R, May CA et al. Toxicity study of erucylphosphocholine in a rat model. Curr Eye Res 2005; 30: 813-820.
16 Thaler S, Choragiewicz TJ, Rejdak R, Fiedorowicz M, Turski WA, Tulidowicz-Bielak $\mathrm{M}$ et al. Neuroprotection by acetoacetate and beta-hydroxybutyrate against NMDAinduced RGC damage in rat-possible involvement of kynurenic acid. Graefes Arch Clin Exp Ophthalmol 2010; 248: 1729-1735.

17 Thaler S, Voykov B, Willmann G, Fiedorowicz M, Rejdak R, Gekeler $\mathrm{F}$ et al. Tempol protects against intravitreous indocyanine green-induced retinal damage in rats. Graefes Arch Clin Exp Ophthalmol 2012; 250: 1597-1606.

18 Naka KI, Rushton WA. S-potentials from luminosity units in the retina of fish (Cyprinidae). J Physiol 1966; 185: 587-599.

19 Fischer MD, Huber G, Beck SC, Tanimoto N, Muehlfriedel R, Fahl $\mathrm{E}$ et al. Noninvasive, in vivo assessment of mouse retinal structure using optical coherence tomography. PLoS One 2009; 4: e7507.

20 Knott EJ, Sheets KG, Zhou Y, Gordon WC, Bazan NG. Spatial correlation of mouse photoreceptor-RPE thickness between SD-OCT and histology. Exp Eye Res 2011; 92: 155-160.

21 Wollensak G, Spoerl E, Wirbelauer C, Pham DT. Influence of indocyanine green staining on the biomechanical strength of porcine internal limiting membrane. Ophthalmologica 2004; 218: 278-282.

22 Takayama K, Sato T, Karasawa Y, Sato S, Ito M, Takeuchi M. Phototoxicity of indocyanine green and brilliant blue $\mathrm{G}$ under continuous fluorescent illumination on cultured human retinal pigment epithelial cells. Invest Ophthalmol Vis Sci 2012; 53: 7389-7394.

23 Creuzot-Garcher C, Acar N, Passemard M, Bidot S, Bron A, Bretillon L. Functional and structural effect of intravitreal indocyanine green, triamcinolone acetonide, trypan blue, and brilliant blue g on rat retina. Retina 2010; 30: 1294-1301.

24 Fukuda K, Shiraga F, Yamaji H, Nomoto H, Shiragami C, Enaida $\mathrm{H}$ et al. Morphologic and functional advantages of macular hole surgery with brilliant blue G-assisted internal limiting membrane peeling. Retina 2011; 31: 1720-1725.

25 Baba T, Hagiwara A, Sato E, Arai M, Oshitari T, Yamamoto S. Comparison of vitrectomy with brilliant blue $\mathrm{G}$ or indocyanine green on retinal microstructure and function of eyes with macular hole. Ophthalmology 2012; 119: 2609-2615.

26 Schuettauf F, Haritoglou C, May CA, Rejdak R, Mankowska A, Freyer $\mathrm{W}$ et al. Administration of novel dyes for intraocular surgery: an in vivo toxicity animal study. Invest Ophthalmol Vis Sci 2006; 47: 3573-3578.

27 Kudo H, Nakazawa T, Shimura M, Takahashi H, Fuse N, Kashiwagi $\mathrm{K}$ et al. Neuroprotective effect of latanoprost on rat retinal ganglion cells. Graefes Arch Clin Exp Ophthalmol 2006; 244: 1003-1009.

28 Manabe S, Lipton SA. Divergent NMDA signals leading to proapoptotic and antiapoptotic pathways in the rat retina. Invest Ophthalmol Vis Sci 2003; 44: 385-392.

29 Kikuchi M, Tenneti L, Lipton SA. Role of p38 mitogenactivated protein kinase in axotomy-induced apoptosis of rat retinal ganglion cells. J Neurosci 2000; 20: 5037-5044.

30 Schuettauf F, Zurakowski D, Quinto K, Varde MA, Besch D, Laties A et al. Neuroprotective effects of cardiotrophin-like cytokine on retinal ganglion cells. Graefes Arch Clin Exp Ophthalmol 2005; 243: 1036-1042.

31 Luke C, Luke M, Sickel W, Schneider T. Effects of patent blue on human retinal function. Graefes Arch Clin Exp Ophthalmol 2006; 244: 1188-1190.

32 Maia M, Margalit E, Lakhanpal R, Tso MO, Grebe R, Torres $\mathrm{G}$ et al. Effects of intravitreal indocyanine green injection in rabbits. Retina 2004; 24: 69-79. 
33 Sekiryu T, Iida T. Long-term observation of fundus infrared fluorescence after indocyanine green-assisted vitrectomy. Retina 2007; 27: 190-197.

34 Rodrigues EB, Penha FM, Farah ME, de Paula Fiod CE, Maia M, Dib E et al. Preclinical investigation of the retinal biocompatibility of six novel vital dyes for chromovitrectomy. Retina 2009; 29: 497-510.

35 Jackson TL, Griffin L, Vote B, Hillenkamp J, Marshall J. An experimental method for testing novel retinal vital stains. Exp Eye Res 2005; 81: 446-454. 